\title{
Development of the Beam-forming Control Device for the Ultrasound Measurement
}

\author{
Hyo-jin Lee ${ }^{1,1}$, Yong-tae Kim and Sun-hyung Kim ${ }^{1}$ \\ Department of Information \& Communication Engineering, Soonchunhyang \\ University, Asan, Chungnam 336-745, Republic of Korea, Korea Research Institute of \\ Standards and Science, 1Doryong-Dong, Yuseong-gu, Daejoen, Republic of Korea. \\ dkid33@naver.com,ytkim@kriss.re.kr,shkim@sch.ac.kr
}

\begin{abstract}
In this thesis, it is presented the proposed development of the Switching MUX with the array transducer by use of Delay-And-Sum Beam-forming technology for a more accurate measurement. Among the several measurement methods, it is studied two ways, i.e. PulseEcho method and the radiation conductance measurement. In this paper, we propose to the multi-purpose Switching MUX all interfacing the two methods mentioned earlier. And it is verified that beam-forming control of the proposed equipment is smoothly implemented through simulation. And multi-purpose Switching MUX proposed in this thesis is modularized by 64ports in order to reduce the hardware size.
\end{abstract}

Keywords: Ultrasound, Array Transducer, Beam-forming, Switching MUX.

\section{Introduction}

This thesis proposes a switching MUX is capable of interfacing with various measurement methods using ultrasound. Measurement using ultrasound is used the measure ultrasound intensity and the thickness of the reflector. In this paper, we propose a switching MUX that can be used in two types of measurement method. In order to use an array transducer, each element is needed delay[1]. Switching MUX provide the delay. In order to use an array transducer, a device for generation a delay for each measurement method was necessary. Switching MUX is provides the interface that can use in a variety of measurement methods. Therefore, it is can be applied to more than one measurement method in a single device. And Switching MUX proves that simulation can be using through. The ultrasound is the sound of more than $20,000 \mathrm{~Hz}$ frequency. Frequency represents how many times the vibration wave in one second. And unit uses $\mathrm{Hz}($ hertz)[2]. Ultrasound of frequency higher than $1 \mathrm{MHz}$ has directivity. In other words, it is emitted in a certain direction as the light. There are four properties of the ultrasound. The first is attenuation. As the distance increase, the intensity of the ultrasound wave decrease. The second is the reflection. When the ultrasound wave enters the reflector, it is the phenomenon in which a part is returned is returned. The third is a property called refraction. Phenomenon that the direction of the beam changes according to the angle of the reflector is refracted. The last is scattering. Irregular reflector is hit by ultrasound. At this time, the ultrasound beam is divided into various directions. This phenomenon is scattering. Ultrasound is an important diagnostic and treatment methods that can be very easily performed over the entire areas of the human body[3]. In addition, it is typical method for non-destructive insertion. Therefore utilization is increasingly. Ultrasound diffusion, absorption, since it is attenuated by scattering, etc., it is required for proper use of the frequency in accordance with the site or application. Ultrasound transducer is generated ultrasound by the piezoelectric element. And it is made from crystal, tourmaline, ceramic, etc.

${ }^{1}$ Corresponding author : Tel.: +82-41-530-1344; Fax:+82-41-530-1548 
Electricity generated by ultrasound is applied to the surface. When an electrical signal is applied to piezoelectric element, it vibrates. Ultrasound measuring device generates ultrasound wave by adding electrical signal to the transducer. And detects the electrical signals generated by the reflected wave coming back[4]. This thesis proposes a Switching MUX which is capable of interfacing with various measurement methods using ultrasound. And it is suggested the structure of the Switching MUX so that it may have variety of measurement method using the ultrasound. And designed to be able to control beam-forming of the proposed equipment is smoothly implemented through simulation. Also implement the Delay-And-Sum beam-forming through simulation. And it makes the modularization in order to reduce the hardware size and to change easily the port control. This thesis is comprised of following chapters. Chapter 1: introduction, Chapter 2: ultrasound measurement method, Chapter 3: array transducer, Chapter 4: beam-forming, Chapter 5: simulation of Delay-AndSum beam-forming, Chapter 6: simulation of switching MUX and is finalized with Chapter 7.

\section{Ultrasound Measurement Method}

Measurement method using ultrasound waves is varied. In this paper, we use two types of measurement method. The first is Pulse-Echo method, it is a method for measuring the thickness of the reflector. And the second is a Radiation conductance measurement which is measures the intensity of the ultrasound waves.

\subsection{Pulse-Echo Method}

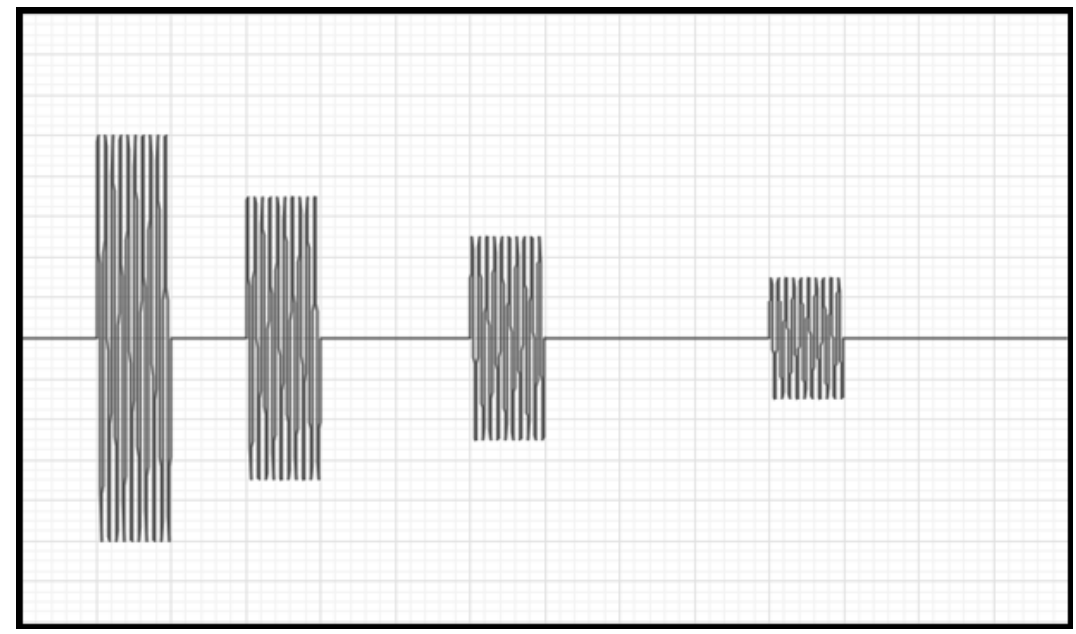

Figure 1. Received Waveforms of Pulse-Echo Method

Method of measuring the thickness of the reflector is Pulse-Echo method to using ultrasound wave. Enter a short pulse signal to the ultrasound transducer. As a result, the ultrasound waves are generated by the piezoelectric effect of the piezoelectric element. When the generated ultrasound wave reaches the reflector, reflection occurs. Reflected ultrasound wave is again arriving to the transducer. Components of the reflected ultrasound waves there are various. One is a wave that has been reflected by the surface of the reflector. And there is such a wave that has been reflected at the bottom of the reflector. Received echo waveform, the shorter the distance to be reflected, it is present near the generated pulse signal. In other words, the distance between the first echo wave and the generated pulse wave is the distance to the reflecting surface. The next received echo waveform is waveform reflected by standing on the bottom of the reflector. When calculating the time difference of the two echo waveform, it is possible to measure the thickness of the reflector. 


\subsection{Radiation Conductance Measurement}

Ultrasound wave is generated different per volt along the ultrasound transducer. Radiation conductance measurement is a method of measuring the intensity of the ultrasound waves $[5,6]$. This method is to position the transducer is facing up from the floor. And position the target fabricated with a material which absorbs ultrasound waves in the upper. The target is connected to the capable of measuring with high precision scales. To produce the ultrasound by enter the pulse signal to the transducer in this state. As a result, the target is rise due to ultrasound forces. When compared with the weight before the shoot the ultrasound waves, it is possible to calculate the intensity of the ultrasound waves. It is comparing the ultrasound performance using the data generated in this way.

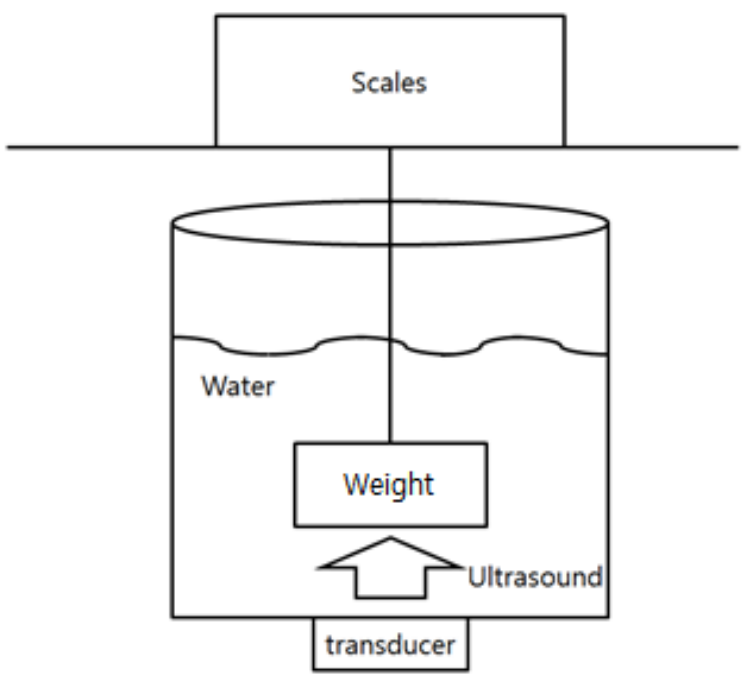

Figure 2. Structure of Radiation Conductance Measurement

\section{Array Transducer}

Ultrasound transducer is generally formed of a single piezoelectric element. Therefore the shape of the ultrasound beam is always constant. The structure of the array transducer is a little different with single ultrasound transducer. The array transducer consists of a piezoelectric element and several of elements. In order to make variable shape of the ultrasound beam, it needs the Switching MUX. Therefore it is possible to adjust the ultrasound delay of an array transducer. So we can change the shape of the beam. Each element of the array transducer is able to vary the release time of the ultrasound wave[7]. Thus, the change the shape of the ultrasound beam means the beam-forming. The type of the beam-forming is various things. In this paper, the Delay-And-Sum beam-forming is used.

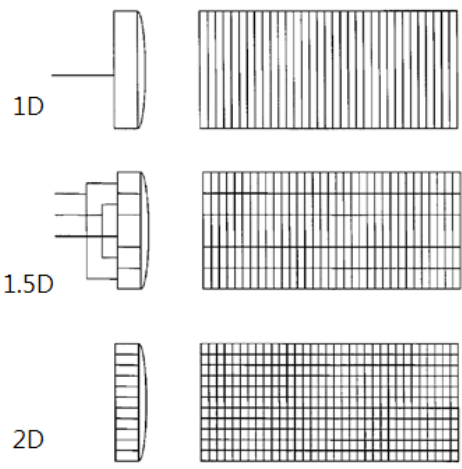

Figure 3. Types of Array Transducer 


\section{Beam-forming}

As mentioned earlier, beam-forming is intended to change the shape of beam. Principle of changing ultrasound beam shape, it is as in the following. At first, ultrasound waves are emitted with a different delay. Emitted ultrasound waves will be subjected to interference with each other. Thus, ultrasound is amplified together, attenuation or offset[8]. Because of this phenomenon, it is possible to focus the ultrasound beam to the desired point.

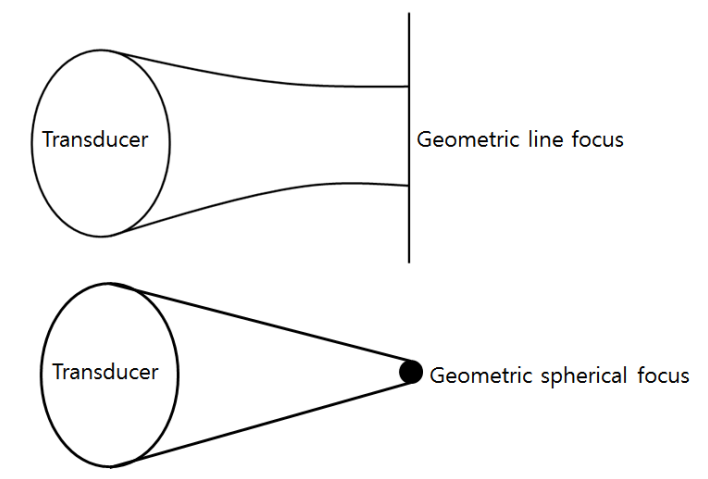

Figure 4. Line Focus and Spherical Focus of Beam-forming

\subsection{Delay-And-Sum Beam-forming}

Delay-And-Sum beam-forming is a beam-forming method using the delay. This method has two types. It is the Tx beam-forming and Rx beam-forming. Tx beam-forming is a beamforming method for delaying at the transmitting end. On the other hand, Rx beam-forming is a method that delaying the received signal. In this paper, discuss the Tx beam-forming. As mentioned earlier, array transducer is present with the array elements on the single transducer to generate ultrasound. Eventually, it is the element that emits ultrasound. In this case, element have the different delay. This makes it possible to change the ultrasound beam into a desired shape $[9,10]$.

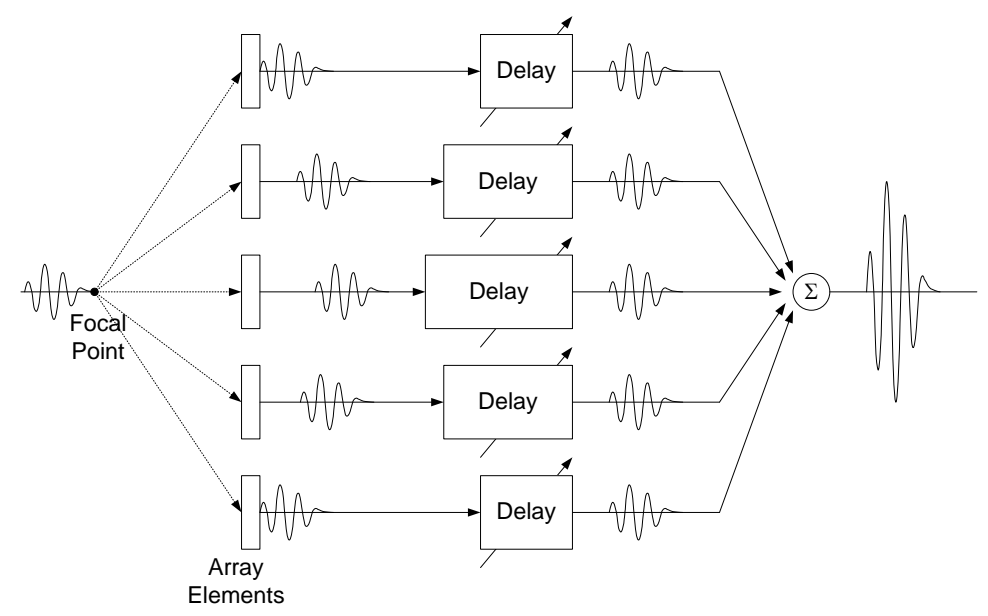

Figure 5. Principle of Delay-And-Sum Beam-forming 


\section{Simulation of Delay-And-Sum Beam-forming}
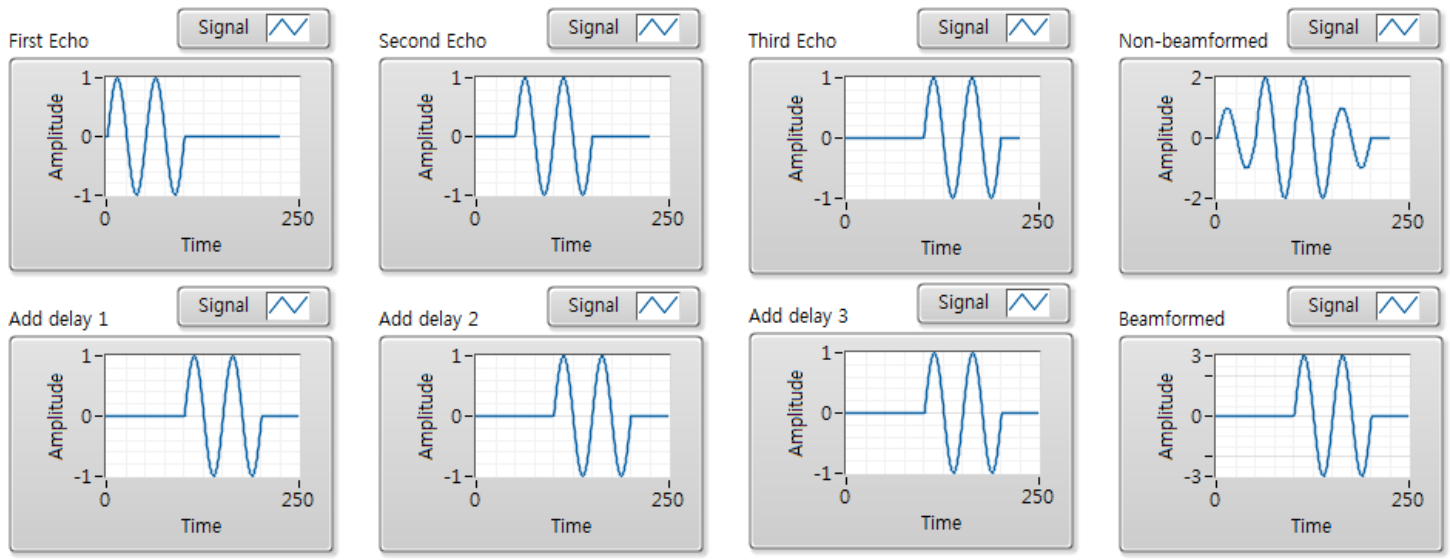

Figure 6. Simulation of Delay-And-Sum Beam-forming

Delay-And-Sum beam-forming is a method for beam-forming by adjusting the delay of the ultrasound waves with different delays. The Figure 6 shows the simulation result of the nonbeam-forming pulse and the beam-forming pulse. The sum of the pulse having a different delay is that the shape is not perfect. So Delay-And-Sum beam-forming adjust the delay of pulses with different delay. The shape of the required beam is formed as a personalized delay of each other. By adjusting the delay of each other, it is made the shape of the desired beam.

\section{Simulation of Switching MUX}

As mentioned earlier, there is a need for a device that proposes the delay for beam-forming of the array transducer. This thesis provides a device that will create a delay. Thus, this paper proposes the variable channel Switching MUX that can be used in Pulse-Echo method and Radiation conductance measurement. The basic operation of Switching MUX would make generate the delay. Using a delay device in Switching MUX, it switches connection port and generates a delay. And a slot type MUX module is made by the LabVIEW software. Therefore, it is possible to use selectively only the required channels.
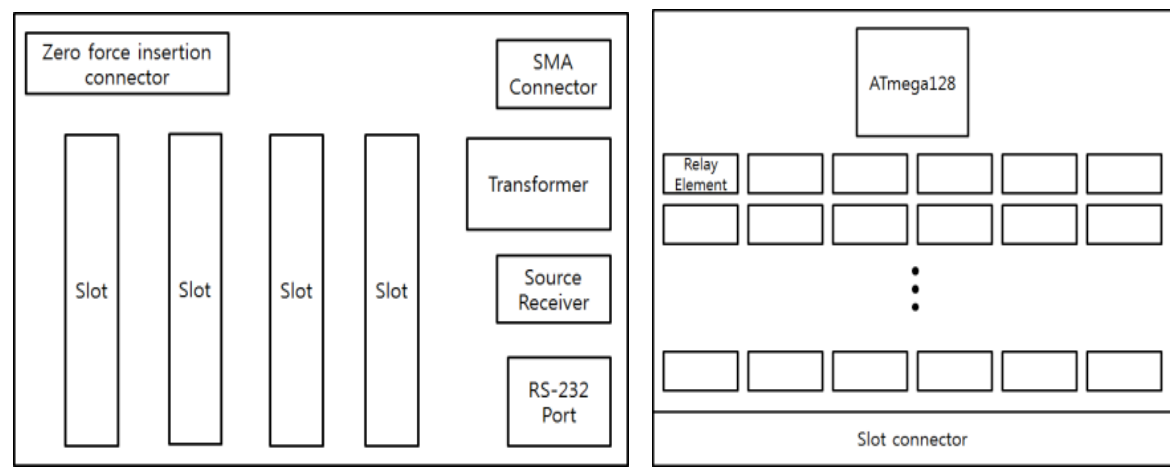

Figure 7. A Block Diagram of Integrated Hardware and 64 Port Module 


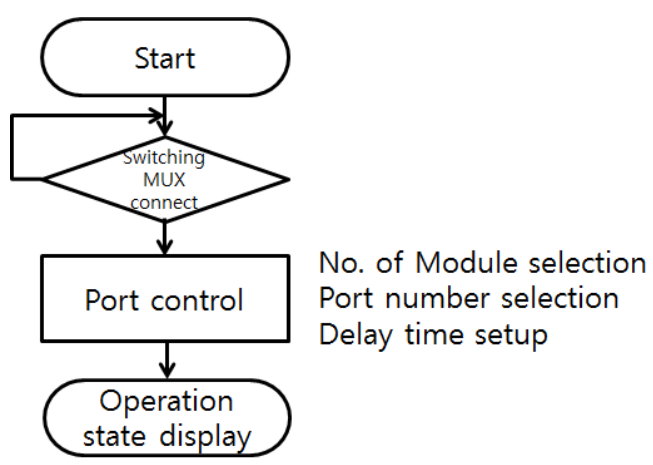

Figure 8. Software Flowchart of Switching MUX

\subsection{Channel Control}

Switching MUX is made modularized by 64 ports, it control only the required ports. Therefore, this chapter haves simulated a 64 channel. The figure 9 shows the basic operation of the channel control. Enter the number of ports that control to when you click the [Open] button, the port is open. When you click the [Close] button, Close the port.

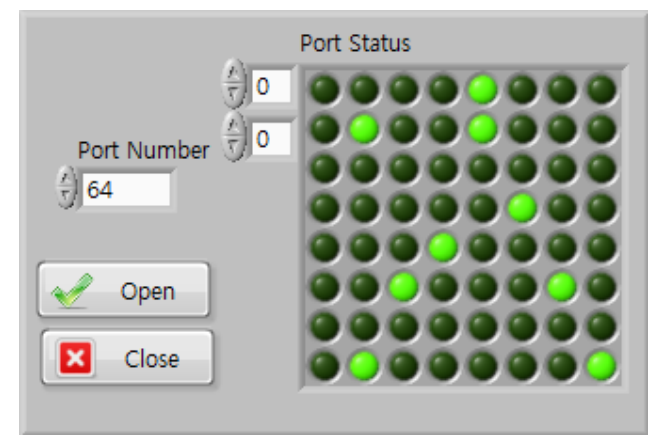

\section{Figure 9. Simulation of a 64 Channel Control}

\subsection{Channel Delay Control}

To use the beam-formed ultrasound using the array transducer, it is necessary to delay a short time to array transducer of each element. To the beam-forming use LabVIEW to add a function that can provide a delay in microseconds.
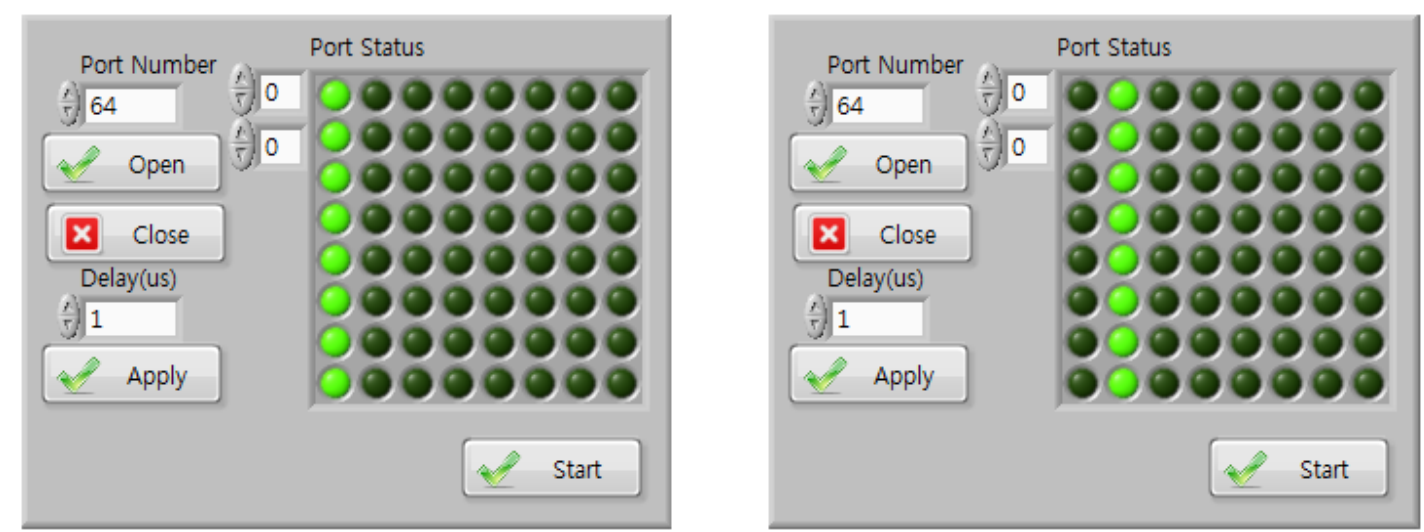

Figure 10. Simulation of the Delay Control of 64 Channels 
The Figure 10 is a simulation of the port Control with additional delays. After you enter the delay time by after entering the port first, and press the [Apply] button. And clicking the [Start] button, and operates based on the delay time of each port is entered. Using the Array Transducer in the above program, it is possible to beam-forming.

\subsection{Additional Modules}

By modularizing the 64 channel ports, and is fabricated to be able to increase the number of channels. If the number of [Number of module] to enter the number of modules of the more users use, it is possible to control the module just entered. Number of port the added modules to start from 65. If enter the three, starting from 129.

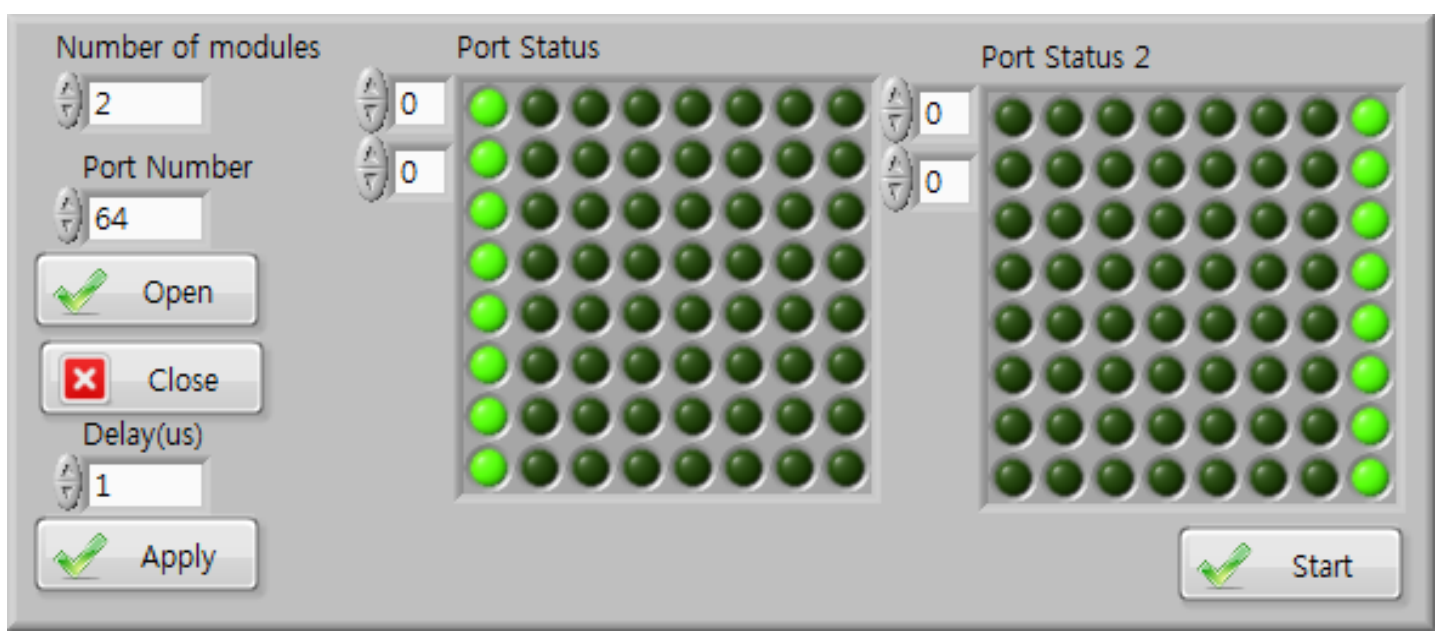

Figure 11. Simulation of Additional Modules

\section{Conclusion}

This thesis provides an interface device which can be used to two ultrasound measurement method. The name of the device is defined Switching MUX is to add ultrasound delay to each element of the array transducer. It was based simulation of Delay-And-Sum beam-forming. And it makes the modularization in order to reduce the hardware size and to change easily the port control. It consists of a programming that can control the 256 ports using the LabVIEW software. It Programming up to four modules. In addition, it was made to be able to provide a delay for each element. Simulation results, it each is possible to provide a delay for each port, it was confirmed that up to four modules can be used. And it is verified that beam-forming control of the proposed equipment is smoothly implemented through simulation. And multi-purpose Switching MUX proposed in this thesis is modularized by 64 ports in order to reduce the hardware size. In the future, it is planned to implement the interface device for the 256 channels by constructing the hardware and to measure the thickness and intensity by using the ultrasound beam-forming with a delay.

\section{References}

[1] M. H. Bae, "Focusing delay calculation method for real-time digital focusing and apparatus adopting the same", U.S. Patent 5,836,881, (1998).

[2] P.D. Edmonds, Ultrasonic, Academic Press, Inc., (1981).

[3] D., and C.R. Johnson, "Methods for procedures related to the electrophysiology of the heart", U.S. Patent 5,529,067, (1995).

[4] K. Erikson, A. Hairston, A. Nicoli,, J. Stockwell, " $123 \times 123 \mathrm{~K}(16 \mathrm{~K})$ ultrasonic transducer hybrid array", Acoust. Imaging, vol. 23, (1997), New York.

[5] D. Y. Yun, M. J. Jho, Y. T. Kim, J. K. Kim, and M. H. Lee, "Design of the electro-acoustic radiation conductance measurement system for medical ultrasonic array probe", IEICE Electronics Express, vol.8, no.12, (2011), pp. 978-983. 
[6] Y. H. Yun, M. J, Jho, Y. T. Kim, and M. H. Lee, "Testing of a Diagnostic Ultrasonic Array Probe and Estimation of the Acoustic Power Using Radiation Conductance", IEEE Sensor Journal, vol.12, no.5, (2012)

[7] W. Frederick, Kremkau, "Diagnostic Ultrasound Principles and Instruments Six edition", W.B. Sauunders Company, (2002).

[8] T. L. Szabo, "Diagnostic ultrasound imaging: inside out", Elselvier academic press, San Diego (2004).

[9] M. H. Bae, "Beamforming Technology in Medical Ultrasound System", Journal of the korean society for nondestructive testing, vol. 32 , no.5, (2012).

[10] J. A. Jensen, "Simulating arbitrary-geometry ultrasound transducers using triangles", Proc. IEEE Ultrason. Symp., vol. 2, (1996) , pp. 885-888.

[11] D. Lipschutz, “Time multiplexed digital ultrasound beamformer”, U. S. Patent 5,469,851, (1998).

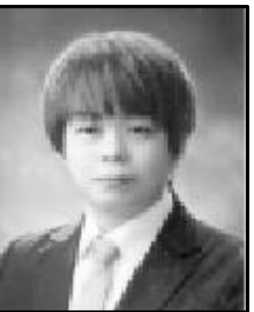

Hyo-jin Lee. Received B.S., degrees in department of Information and Communication Engineering from Soonchunhyang University, korea, in 2014.

March 2014- the present: Department of Information and Communication Engineering, Soonchunhyang University, M. S. candidate.

His research interests include Ultrasound, u-health, Sensor network.

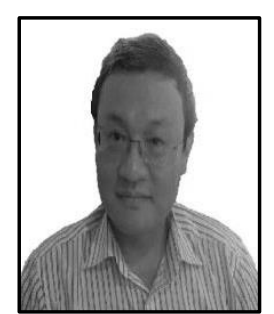

Yong-tae Kim. Received B.S, M.S, and Ph.D. degrees in department physics from Hankuk University of Foreign Studies. South korea, in 1986, 1988 and 1997, respectively.

September 1997- present: senior research scientist in medical Metrology Center, Korea Research Institute of Standard and Science, March 2006- present: Professor in Department of Medical physics, University of Science and Technology.

His research interest includes Medical ultrasound and related metrology.

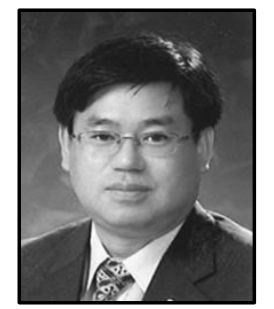

Sun-Hyung Kim. Received his B. S., M. S., and Ph.D. degrees in Electronic Engineering from Sungkyunkwan University, Korea, In 1979, 1981 and 1988, respectively. Since 1989, He has been a professor in Department of Information and Communication Engineering, Soonchunhyang University.

From 2005 to the Present, he was a vice-chairman of Korea University Invention Association. From 2013 to the Present, he was a vice-chairman of Korea Institute of Information Technology.

His research interests include Data Communication, Embedded system, Network, etc. 